\title{
Utilizando la metodología fitosociológica para diferenciar hábitats: un ejemplo con especies chilenas de Schinus (Anacardiaceae) en la Región de Aisén, Chile
}

\author{
Using plant sociological methods to differentiate habitats: an example with Chilean \\ species of Schinus (Anacardiaceae) in the Aisen Region, Chile
}

\author{
Carlos Ramírez ${ }^{1 *}$, Víctor Sandoval ${ }^{2}$, Juan Carlos Cisternas ${ }^{3}$, Hugo Rivera $^{3}$, Osvaldo Vidal ${ }^{4}$, \\ Cristina San Martín ${ }^{5}$, Miguel Álvarez ${ }^{6} \&$ Yéssica Pérez ${ }^{5}$
}

${ }^{1}$ Departamento de Ecología, Facultad de Ciencias Biológicas, Pontificia Universidad Católica de Chile, Alameda 340, Santiago, Chile.

${ }^{2}$ Laboratorio de Geomática, Facultad de Ciencias Forestales y Recursos Naturales, Universidad Austral de Chile, Campus Teja Avenida Zoltan Nagy Yansky s/n, Valdivia, Chile.

${ }^{3}$ Corporación Nacional Forestal de Chile, km 2, ruta 240, Coihaique, Aisén, Chile y Av. Bulnes 285, Santiago, Chile.

${ }^{4}$ Instituto de La Patagonia, Departamento de Botánica, Universidad de Magallanes, Av. Bulnes 01855, Punta Arenas, Chile.

${ }^{5}$ Instituto de Ciencias de la Tierra, Facultad de Ciencias, Universidad Austral de Chile, Campus Teja, Av. Rector Eduardo Morales Miranda, Edificio Dr. Emilio Pugín Ríos s/n, Valdivia, Chile.

${ }^{6}$ INRES-Vegetationsökologie, Rheinische-Friedrich-Wilhelms-Universität, Nussalle 9, D-53115 Bonn, Deutschland.

*cramirez@uach.cl

\section{RESUMEN}

Se estudiaron los hábitats de Schinus patagonicus y Schinus marchandii en la Patagonia chilena, con el propósito de determinar las comunidades vegetales en que crecen y el carácter primario o secundario de ellas, para ayudar a establecer el estado de conservación de las dos especies. Se trabajó con metodología fitosociológica para caracterizar florística y vegetacionalmente las comunidades. La tabla de vegetación se ordenó con métodos tradicionales, utilizando especies diferenciales y estadísticos multivariables de clasificación y ordenación. La tabla fitosociológica inicial presentó 68 especies vegetales siendo 26 de ellas malezas introducidas. En el espectro biológico predominaron hemicriptófitos y con menor importancia, fanerófitos y terófitos. La ordenación tradicional separó las asociaciones Mutisio-Schinetum patagonicae (matorral de clavel del aire y molle) y Colliguajo-Schinetum marchandii (matorral estepario de duraznillo y laura) que se describen como nuevas. La comparación de estas dos comunidades permitió establecer que son florísticamente muy diferentes y que su afinidad fitosociológica es baja: la primera es una comunidad de matorral secundario que reemplaza al bosque de lenga y al matorral de ñire caducifolios degradados por la acción humana y la segunda, una comunidad esteparia primaria. La abundante presencia de Mulinum spinosum permite diferenciar estadios degradados para la primera y esteparios extremos para la segunda. El carácter secundario de la comunidad de clavel del aire y molle confirma la mayor agresividad y capacidad de competencia de Schinus patagonicus, lo que asegura su sobrevivencia como especie, pero el carácter primario y el pequeño tamaño del área del matorral de duraznillo y laura, además de la reducción de tamaño de Schinus marchandii, indican que el estado de conservación de esta última especie en Chile es precario.

Palabras clave: Vegetación, comunidades, clasificación, ordenación, Patagonia chilena.

\begin{abstract}
Habitats of Schinus patagonicus and Schinus marchandii in the Chilean Patagonia were studied, in order to determine the plant communities into these species growing and the primary or secondary character of them, to help establish the conservation status of the two species. We worked with phytosociological methods to characterize floristic and vegetationaly the communities. The vegetation table is ordered with traditional methods, using differential species and with multivariate statistical classification and ordination methods. The initial vegetation table provided 68 plant species of which 26 are introduced weed. In the biological spectrum dominated hemicryptophytes and minor phanerophytes and therophytes. Traditional management of the vegetation table separated the Mutisio-Schinetum patagonicae ("clavel del aire" and "molle" scrub) and Colliguajo-Schinetum marchandii ("duraznillo" and "laura" steppe scrub) new associations
\end{abstract}


that are described. Comparison of these two communities established that they are floristically very different and that their phytosociological affinity is low, the first is a secondary scrub community that replaces the deciduous patagonic "lenga" forest and the "ñire" scrub degraded by human action and the second, a primary steppe community. The abundant presence of Mulinum spinosum differentiates degraded stages of these two communities specially the first. The secondary character of the "clavel del aire" and "molle" community confirms the more aggressive and competitive position of Schinus patagonicus, which ensures their survival as a species, but the primary character and the small size of the scrub of "laura" and "duraznillo" scrub also areal reduction of Schinus marchandii indicate that the conservation status of the latter species in Chile is poor.

KEYwords: Vegetation, communities, classification, ordination, Chilean Patagonia.

\section{INTRODUCCIÓN}

Según Barkley (1957) el género Schinus perteneciente a la Familia Anacardiaceae presenta 9 especies en Chile: Schinus kauselii F.A. Barkley, Schinus latifolius (Gillies ex Lindl.) Engl., Schinus marchandii F.A. Barkley, Schinus molle (L.) DC. var. rusbyi F.A. Barkley, Schinus montana Engl., Schinus patagonicus (Phil.) I.M. Johnst, Schinus pearcei Engl., Schinus polygama (Cav.) Cabrera y Schinus velutinus (Turcz.) I.M. Johnst., todas integrantes de bosques y matorrales esclerófilos de Chile central (Cabrera 1938, Barkley 1944, Reiche 1898, Muñoz 1966), no obstante dos de ellas: Schinus marchandii y Schinus patagonicus avanzan hacia el sur a través de la diagonal árida que se extiende desde el desierto de Atacama hasta la costa de la Patagonia oriental en Argentina, para instalarse en la Patagonia chilena, específicamente en la Región de Aisén (Isla et al. 2003, Saldivia \& Rojas 2008, Villagrán \& Hinojosa 1997). La última monografía de la familia Anacardiaceae para Chile (Rodríguez 2011) reduce el número de especies, pero mantiene estas dos especies que llegan a la Patagonia. De ninguna de estas dos especies se conocen los hábitats, ni su estado de conservación en Chile, aunque en Argentina ambas son abundantes. No obstante para Schinus marchandii, la Corporación Nacional Forestal de Chile está actualmente proponiendo su inclusión preliminar en la categoría: En peligro crítico, dado los antecedentes existentes (CONAF 2012, Ministerio del Medio Ambiente 2013). Se espera que este trabajo sirva para apoyar esta propuesta, al dar a conocer las características de los ambientes (hábitats) en que sobrevive y el estado de conservación de ellos.

Aunque las dos especies de Schinus de Aisén se diferencian claramente por su morfología, ambas reciben el nombre común de laura o molle, entre otros, por lo que utilizaremos mejor los correspondientes nombres científicos. S. patagonicus es un arbusto frondoso de hasta $6 \mathrm{~m}$ de altura, inerme, es decir, sin espinas, con hojas grandes, aovadas, de superficie foliar mayor que $4 \mathrm{~cm}^{2}$, de ápice agudo y borde crenado. Inflorescencia terminal muy ramificada, multiflora, con pedúnculos largos y con frutos abundantes que sobresalen de entre las hojas. Por el contrario, S. marchandii es un arbusto ralo, poco frondoso, de hasta $3 \mathrm{~m}$ de alto, espinoso con espinas de origen caulinar, con pocas hojas espatuladas, de pequeño tamaño y superficie $\left(<\mathrm{de} 4 \mathrm{~cm}^{2}\right)$, de ápice agudo a romo y con borde liso. Inflorescencias laterales, paucifloras de pedúnculos cortos y ramificados, cubiertas por las hojas. Produce pocos frutos (Tabla I). Por relatos de lugareños esta última especie formaba, en el pasado, arbolitos de hasta $6 \mathrm{~m} \mathrm{de}$ altura, los que fueron cortados para combustible doméstico. Según el conocimiento empírico de los vecinos de los lugares estudiados $S$. marchandii estaría asociado a ambientes más xéricos y esteparios, mientras que $S$. patagonicus sería propio de ambientes más húmedos y primitivamente boscosos. El objetivo del presente estudio es tratar de diferenciar los hábitats de ambas especies, homologando éstos a la comunidad (asociación) vegetal a la que pertenece cada una, suponiendo que deberían ser muy diferentes. Para ello se utilizan metodologías de la fitosociología o sociología vegetal, poderosas herramientas para describir el paisaje determinando unidades fisonómicas, diferenciadas por la proporción de formas de vida o de crecimiento (espectro biológico), conocidas como formaciones vegetales (Dengler et al. 2008) y las subdivisiones de ellas, las asociaciones vegetales, diferenciadas por su composición florística (Mueller-Dombois \& Ellenberg 1974, Knapp 1984), estas últimas han sido utilizadas con éxito por las CCEE para nombrar los hábitats a proteger (Comunidades Europeas 1992) y recientemente, por Ramírez et al. (2009) para detectar especies vegetales con problemas de conservación.

Las formaciones vegetales corresponden a unidades del paisaje vegetal diferenciales a simple vista, tales como bosques, matorrales, praderas, estepas, entre otras; en cambio determinar y diferenciar las asociaciones vegetales implica conocer la diversidad florística, métodos de muestreo y de tabulación, separación de especies diferenciales y metodología estadísticas multivariadas de clasificación y ordenación (Ramírez et al. 1997). Tanto las formaciones como las asociaciones vegetales pueden tener un carácter primario, secundario o incluso terciario, según la posición que ocupen en la dinámica de degradación antropogénica de la vegetación, las primarias son originales del lugar y generalmente están formadas casi exclusivamente por plantas nativas, mientras que las otras representan estadios de diferente intervención humana, en las cuales abundan especies vegetales introducidas. Series de degradación en la vegetación de Aisén han sido descritas por Ramírez et al. (2012). 
TABLA I. Diferenciación morfológica por hábito de las especies de Schinus de Aisén.

TABLE I. Morphological differentiation by habit Schinus species Aisen.

\begin{tabular}{lcc}
\hline CARACTERíSTICAS /EsPECIES & Schinus patagonicus & Schinus marchandii \\
\hline Forma de crecimiento & Arbusto frondoso & Arbusto ralo \\
Tamaño & Hasta $6 \mathrm{~m}$ & Hasta $3 \mathrm{~m}$ \\
Defensa & Inerme & Espinoso \\
Espinas & Ausentes & Presentes \\
Cantidad de hojas & Muchas & Pocas \\
Forma foliar & Aovada & Espatuladas, variables \\
Ápice foliar & Agudo & Agudo a obtuso \\
Superficie foliar & $>4 \mathrm{~cm}^{2}$ & $<4 \mathrm{~cm}^{2}$ \\
Borde foliar & Crenado & Liso \\
Inflorescencia & Multiflora & Pauciflora \\
Pedúnculos & Largos, ramificados & Cortos, poco ramificados \\
Ubicación de frutos & Terminales & Laterales \\
Posición de frutos & Sobresalen de las hojas & Entre las hojas \\
Cantidad de frutos & Muchos & Pocos \\
\hline
\end{tabular}

\section{MATERIAL Y MÉTODOS}

LUGAR DE TRABAJO

El trabajo se realizó en las comunas de Río Ibáñez (desde

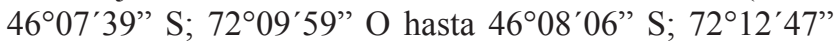

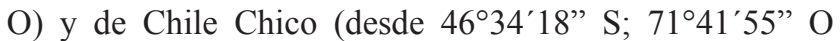
hasta 46³9'31" S; 7141'27" O) de la Región de Aisén del General Carlos Ibáñez del Campo. En esta Región de Chile el relieve, el mar y la cordillera son responsables de una zonificación geográfica que incide directamente en la estructura y composición florística de la vegetación. En un transecto de cordillera a mar se presentan zonas cordilleranas esteparias, zonas intermedias con un relieve muy ondulado y zonas insulares, expuestas al Pacífico (Quintanilla 1989).

Los diagramas climáticos de la Fig. 1 reflejan estas diferentes y contrastantes condiciones (Hajek \& Di Castri 1975). El clima de Puerto Aisén, ciudad ubicada en la zona litoral con predominancia de bosques siempre verdes, se presenta como muy húmedo con exceso de humedad durante todo el año y prácticamente sin meses de sequía. El promedio anual de temperatura alcanza a $9^{\circ} \mathrm{C}$, con medias máximas y mínimas de $12,7^{\circ} \mathrm{C}$ y de $5,6^{\circ} \mathrm{C}$ respectivamente, mientras que la precipitación media anual bordea los $3.000 \mathrm{~mm}$. Por lo anterior, es un clima con escasas variaciones estacionales, típicamente oceánico (Novoa \& Villaseca 1989).

En la ciudad de Coihaique, ubicada más al interior, el clima es más continental seco y extremo, con notorias oscilaciones anuales de la precipitación y la temperatura.
Sólo entre los meses invernales de mayo, junio, julio y agosto se presentan pequeños excesos de humedad, mientras que en verano (diciembre enero y febrero) hay oscilaciones térmicas. La vegetación natural de esta zona corresponde a bosques y matorrales caducifolios. Aunque el promedio mensual anual de temperatura alcanza a $9{ }^{\circ} \mathrm{C}$ igual que en Aisén, sin embargo, las oscilaciones son mayores, alcanzando la media máxima a $12,6^{\circ} \mathrm{C}$ y la mínima a $4,2^{\circ} \mathrm{C}$. La precipitación media anual desciende considerablemente llegando a menos de la mitad de la presentada en Aisén, con $1.164 \mathrm{~mm}$.

En Chile Chico, situado en la ribera sur del lago General Carrera, el clima es típicamente continental con grandes oscilaciones térmicas y pluviales. El diagrama ombrotérmico muestra que la precipitación sólo se hace apreciable en la época invernal entre mayo, junio, julio $\mathrm{y}$ agosto, con un promedio de pluviosidad anual que no supera los $200 \mathrm{~mm}$. Esos meses podrían entonces considerarse húmedos, es decir con mayor precipitación que evaporación, pero los meses restantes son áridos, dado que la curva de temperatura se sobrepone a la de precipitación, indicando que la evaporación supera el agua caída. El promedio térmico anual de $11,5^{\circ} \mathrm{C}$ que supera en $2,5^{\circ} \mathrm{C}$ al de las otras dos ciudades presenta también una mayor oscilación que alcanza a $6,4^{\circ} \mathrm{C}$ anuales, con 11,5 ${ }^{\circ} \mathrm{C}$ de media máxima y $5,1{ }^{\circ} \mathrm{C}$ de media mínima. Amigo \& Ramírez (1998) confirman lo anterior, determinando a los tres climas como pertenecientes al piso supratemperado, pero el ombro tipo sería ultraperhúmedo en Aisén, húmedo 
en Coihaique y seco en Chile Chico. Estas grandes diferencias climáticas, especialmente de precipitación, condicionan las franjas longitudinales de vegetación que se presentan en la Región de Aisén.

De acuerdo a todo lo anterior, la vegetación de la Región de Aisén es susceptible a ser dividida en tres franjas longitudinales paralelas con macroclimas y vegetación muy diferentes (Quintanilla 1989). La primera corresponde a la zona litoral e insular húmeda de archipiélagos con un clima oceánico, con precipitaciones promedios de 3.000 $\mathrm{mm}$ anuales, que posee bosques y matorrales siempre verdes (Teillier \& Marticorena 2002, Ramírez et al. 2004, 2006, Álvarez et al. 2010). La segunda, corresponde a una zona intermedia de valles cordilleranos con dominancia de bosques y matorrales caducifolios y una precipitación que no supera los $500 \mathrm{~mm}$ anuales indicando un clima más continental, con mayor oscilaciones térmicas (Amigo \& Ramírez 1998, Gajardo 1994, Luebert \& Pliscoff 2006, Vidal et al. 2011). La estepa patagónica sería parte de una tercera zona, que se caracteriza por presentar comunidades graminosas, pulviniformes, arbustivas y subarbustivas en un macroclima estepario, seco y temperado, el que suele ser morigerado por la presencia de lagos como sucede en la zona de Chile Chico, donde por la sequía y el calor, incluso aparecen cactáceas en suelos arenosos (Saldivia \& Rojas 2008).

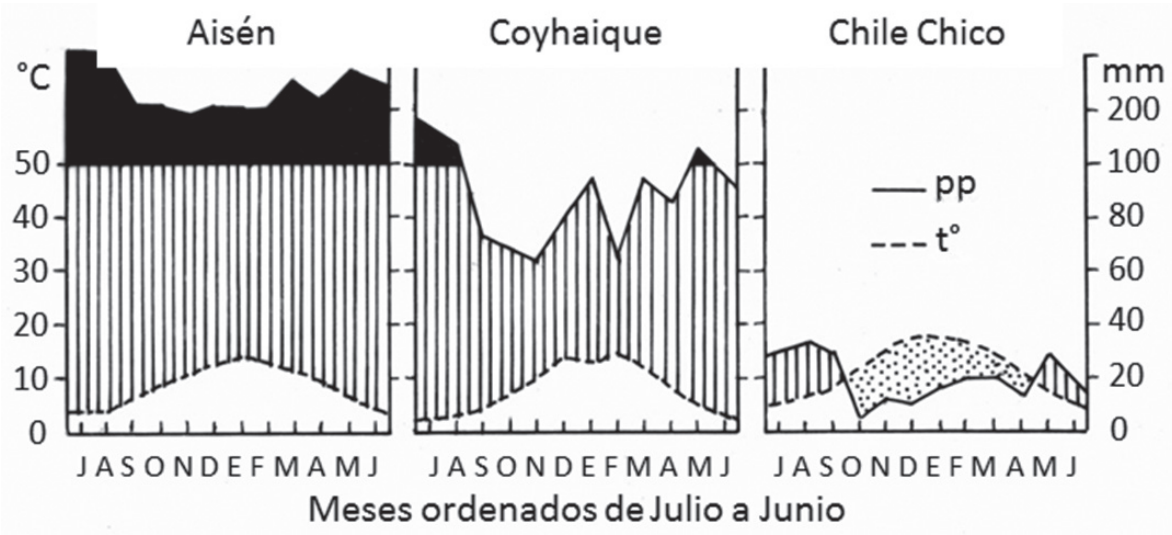

Figura 1. Diagramas climáticos ombrotérmicos de las ciudades de Aisén, Coihaique y Chile Chico en la Región de Aisén del General Carlos Ibáñez del Campo, Chile según Hayek \& Di Castri (1975). Abreviaturas: pp = precipitación promedio mensual, $\mathrm{t}^{\circ}=$ temperatura promedio mensual.

Figure 1. Ombrothermal climate diagrams of the Aisén, Coihaique y Chile Chico in the Aisén Región del General Carlos Ibáñez del Campo, Chile after Di Castri \& Hayek (1975). Abbreviations: $\mathrm{pp}=$ Monthly average rainfall, $\mathrm{t}^{\circ}=$ monthly average temperature.

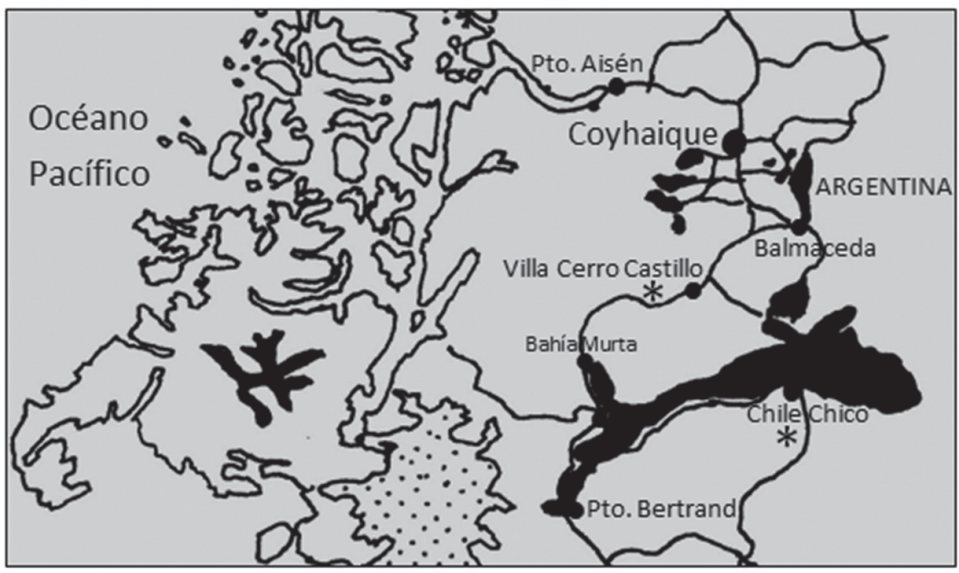

Figura 2. Parte central de la Región de Aisén del General Carlos Ibáñez del Campo, Chile. Los asteriscos indican los lugares estudiados. El que está ubicado al Norte (cerca de Villa Cerro Castillo) corresponde a Comunidades de Schinus patagonicus, el ubicado al Sur (cerca de Chile Chico) a comunidades de Schinus marchandii.

Figure 2. Central part of the Aisen Región of General Carlos Ibanez del Campo, Chile. Asterisks indicate the locations studied. Which is located to the north (near Villa Cerro Castillo) corresponds to Schinus patagonicus communities, located to the south (near Chile Chico) to Schinus marchandii communities. 
Métodos

Previo al inicio del trabajo se prospectó la Región de Aisén en el marco del Proyecto CONAF-UACh "Actualización del Catastro del Bosque Nativo en la Región de Aisén”, encontrándose comunidades claramente diferenciables de las dos especies de Schinus descritas para la región, que se presentan en localidades geográficamente separadas y en hábitats distintos. Por lo anterior, se levantaron censos de vegetación en los respectivos lugares por separado (Fig. 2). En los sectores intermedios no se encontraron las comunidades.

El trabajo de terreno se realizó levantando 20 censos de vegetación con la metodología fitosociológica de la Escuela europea (Mueller-Dombois \& Ellenberg 1974, Braun-Blanquet 1979). Se levantaron 10 censos para cada comunidad utilizando un área de muestreo superior a $200 \mathrm{~m}^{2}$ de acuerdo a las instrucciones de Knapp (1984), anotando la totalidad de la flora presente en la parcela y luego estimando, en porcentaje, la cobertura de los individuos de cada una. Para coberturas bajo $1 \%$ se utilizaron los signos "+" y "r" con su significado tradicional donde el primero indica la presencia de varios individuos y el segundo la presencia de sólo uno de ellos, en todo caso estos signos fueron elevados a la unidad para cálculos posteriores. La ubicación geográfica de los censos se presenta en la Tabla II.

Con los censos levantados se construyó una tabla fitosociológica inicial de la cual se obtuvo la lista florística cuya nomenclatura fue actualizada en el sitio WEB "theplantlist" del Jardín Botánico de Missouri y cuyo origen fitogeográfico se tomó de Zuloaga et al. (2008). Las formas de vida de las especies vegetales se determinaron con la clave de Mueller-Dombois \& Ellenberg (1974) y con ellas se construyeron espectros biológicos.

La ordenación de la tabla inicial se realizó con metodología tradicional diferenciando grupos de acuerdo a la presencia de especies diferenciales que se excluyen mutuamente (Dierschke 1994), logrando separar dos comunidades que fueron descritas como asociaciones nuevas de acuerdo a las normas de nomenclatura fitosociológica vigentes (Izco \& Del Arco 2003) ya que no se presentaban en la literatura fitosociológica revisada. Se realizó un análisis de conglomerados para clasificar las unidades de muestreo en grupos sintaxonómicos (comunidades vegetales), utilizando el método jerárquico flexible beta con la distancia Bray-Curtis y un parámetro $\beta=-0,25$ (McCune \& Grace 2002). Este procedimiento ha sido recomendado para análisis de vegetación pues entrega resultados semejantes al método de la varianza mínima, pero no ofende el principio de minimización de error de la suma de cuadrados dentro de los grupos y además, conserva las propiedades del espacio (Legendre \& Legendre 1998). También se realizó un Análisis Multidimensional Nométrico (Non metric Multidimensional Scaling) (Clarke
1993, Legendre \& Legendre 1998) para obtener una configuración gráfica de las comunidades, visualizando la relación entre los sitios en función de la composición florística de cada uno. Este método es ventajoso porque examina la estructura de las matrices sin asumir relaciones lineales entre las variables de estudio y porque a través de una iteración, muestra las distancias ordenadas sobre cualquier tipo de transformación o relativización de los datos (McCune \& Grace 2002). El estadístico resultante es un valor denominado Stress, el cual indica cómo la configuración de la gráfica representa las distancias entre los sitios. Idealmente el valor del Stress debe fluctuar entre 10 y 30\% (Roeland \& Coe 2005). Los análisis multivariados se realizaron usando los paquetes estadísticos BiodiversityR (Roeland \& Coe 2005) y MASS v. 7.3 (Venables \& Ripley 2002) mediante la versión R Studio 2.15.1 (R_Development_Core_Team 2012).

Se confeccionaron tablas independientes para las dos comunidades encontradas y en ellas, se calculó un valor de importancia considerando la frecuencia y cobertura relativa de las especies de acuerdo a Wikum \& Shanholtzer (1978), designando además, un censo tipo para cada una (Izco \& Del Arco 2003), proponiéndose los correspondientes nombres para ellas.

Por último se procedió a comparar las dos asociaciones descritas en su flora, origen fitogeográfico y similitud florística con el índice de similitud de Jaccard (Badii et al. 2007) y con el coeficiente de comunidad de Ellenberg (Ramírez et al. 1997) utilizando la cobertura promedio absoluta y ponderada, dividiendo la cobertura total por la frecuencia absoluta y ponderada, es decir, por el número total de censos o sólo por aquellos donde la especie en cuestión estaba presente.

\section{RESULTADOS}

La tabla fitosociológica inicial construida con los censos está formada por 68 especies y 20 censos. Datos de nomenclatura, taxonómicos, de origen fitogeográfico y de formas de vida se presentan en la Tabla III. Las especies con mayor presencia (frecuencia) en ella fueron: Senecio patagonicus presente en 17 censos, Berberis microphylla en 16, Acaena pinnatifida en 14, Mulinum spinosum en 12, Schinus patagonicus y Schinus marchandii en 10 censos respectivamente, todas especies nativas, indicando una segregación de sus hábitats. Presentes en 9 censos aparecieron 3 especies: Colliguaja integerrima Dactylis glomerata y Plantago lanceolata siendo sólo la primera nativa y las otras dos malezas alóctonas. La mayor frecuencia de las plantas nativas indica condiciones extremas de hábitat para la zona estudiada que las malezas no soportan. 
Hábitats de especies de Schinus en Aisén: Ramírez, C. ET AL.

TABLA II. Ubicación geográfica (coordenadas IGM) y fecha de los censos de vegetación levantados. Se indica la comunidad a la que pertenece cada relevé.

TABLE II. Geographic location (coordinates IGM) and date of the vegetation samples. Community to which each relevé belongs is indicated.

\begin{tabular}{ccccc}
\hline CoMUNIDAD & CENSO & FECHA & CoORDENADAS IGM (X) & $\begin{array}{c}\text { CoORDENADAS } \\
\text { IGM (Y) }\end{array}$ \\
\hline Mutisio-Schinetum patagonicae & 1 & $18-01-2011$ & 718851 & 4887946 \\
& 2 & $18-01-2011$ & 718851 & 4887946 \\
& 3 & $18-01-2011$ & 718873 & 4887822 \\
Colliguajo-Schinetum marchandii & 4 & $18-01-2011$ & 717730 & 4888019 \\
& 5 & $18-01-2011$ & 717097 & 4888316 \\
& 6 & $18-01-2011$ & 717097 & 4888031 \\
& 7 & $18-01-2011$ & 716030 & 4888005 \\
& 8 & $18-01-2011$ & 715996 & 4887238 \\
& 9 & $18-01-2011$ & 715321 & 4887218 \\
& 10 & $18-01-2011$ & 715294 & 4838841 \\
& 12 & $21-01-2011$ & & 4838841 \\
& 13 & $21-01-2011$ & 293213 & 4838640 \\
& 14 & $21-01-2011$ & 295336 & 4838522 \\
& 15 & $21-01-2011$ & 293616 & 4836915 \\
& 16 & $21-01-2011$ & 293652 & 4829028 \\
& 17 & $21-01-2011$ & 293887 & 4829028 \\
& 18 & $21-01-2011$ & 293887 & 4829952 \\
& 19 & $21-01-2011$ & 294562 & 4829952 \\
& 20 & $21-01-2011$ & 294724 & 4832344 \\
\hline
\end{tabular}

TABla III. Lista de la flora de las comunidades de Schinus de Aisén. Abreviaturas: Or. = Origen: $\mathrm{N}=$ nativo, $\mathrm{I}=$ introducido; FV= Forma de vida: $\mathrm{F}$ = fanerófito, $\mathrm{C}$ = caméfito, $\mathrm{H}=$ hemicriptófito, $\mathrm{Cr}$ = criptófito, $\mathrm{T}=$ terófito.

TABLE III. List of flora of Schinus communities in Aisen. Abbreviations: Or. = origin: $\mathrm{N}=$ native, $\mathrm{I}=$ introduced, $\mathrm{FV}=\mathrm{Life}$ forms: $\mathrm{F}=$ phanerophyte, $\mathrm{C}$ = chamaephyte, $\mathrm{H}=$ hemicryptophyte, $\mathrm{Cr}=$ cryptophyte, $\mathrm{T}=$ therophyte.

\begin{tabular}{|c|c|c|c|c|}
\hline NOMBRE CIENTÍFICO & FAMILIA & NOMBRE COMÚN & OR. & FV \\
\hline Acaena integerrima Gillies ex Hook. et Arn. & Rosaceae & Cadillo grande & $\mathrm{N}$ & $\mathrm{C}$ \\
\hline Acaena pinnatifida Ruiz et Pav. & Rosaceae & Pimpinela & $\mathrm{N}$ & $\mathrm{H}$ \\
\hline Acaena antarctica Hook. f. & Rosaceae & Amores secos & $\mathrm{N}$ & $\mathrm{H}$ \\
\hline Achillea millefolium $\mathrm{L}$. & Asteraceae & Milenrama & $\mathrm{I}$ & $\mathrm{H}$ \\
\hline Adesmia boronoides Hook. f. & Fabaceae & Paramela & $\mathrm{N}$ & $\mathrm{C}$ \\
\hline Adesmia lotoides Hook. f. & Fabaceae & - & $\mathrm{N}$ & $\mathrm{C}$ \\
\hline Agrostis capillaris L. & Poaceae & Chépica & $\mathrm{I}$ & $\mathrm{H}$ \\
\hline Aira caryophyllea $\mathrm{L}$. & Poaceae & - & I & $\mathrm{T}$ \\
\hline Amaranthus deflexus L. & Amaranthaceae & Moco de pavo & I & $\mathrm{T}$ \\
\hline Anemone multifida Poir. & Ranunculaceae & - & $\mathrm{N}$ & $\mathrm{H}$ \\
\hline Baccharis magellanica (Lam.) Pers. & Asteraceae & Chilca rastrera & $\mathrm{N}$ & $\mathrm{C}$ \\
\hline Baccharis obovata Hook. et Arn. & Asteraceae & Chilca & $\mathrm{N}$ & $\mathrm{F}$ \\
\hline Berberis microphylla G. Forst. & Berberidaceae & Calafate & $\mathrm{N}$ & $\mathrm{F}$ \\
\hline Bromus catharticus Vahl. & Poaceae & Pasto lanco & $\mathrm{N}$ & $\mathrm{H}$ \\
\hline Cerastium arvense L. & Caryophyllaceae & Cuernecillo & $\mathrm{I}$ & $\mathrm{T}$ \\
\hline
\end{tabular}




\begin{tabular}{|c|c|c|c|c|}
\hline NOMBRE CIENTÍFICO & FAMILIA & NomBre COMÚN & OR. & FV \\
\hline Matricaria discoidea DC. (Pursh.) Rydb. & Asteraceae & Manzanilla & I & $\mathrm{T}$ \\
\hline Cirsium vulgare (Savi) Ten. & Asteraceae & Cardo negro & I & $\mathrm{T}$ \\
\hline Colletia hystrix Clos. & Rhamnaceae & Yaqui & $\mathrm{N}$ & $\mathrm{F}$ \\
\hline Colliguaja integerrima Gillies et Hook. & Euphorbiaceae & Lechero & $\mathrm{N}$ & $\mathrm{F}$ \\
\hline Crepis capillaris (L.) Wallr. & Asteraceae & - & I & $\mathrm{T}$ \\
\hline Dactylis glomerata L. & Poaceae & Pasto ovillo & I & $\mathrm{H}$ \\
\hline Discaria chacaye (G. Don) Tortosa & Rhamnaceae & Chacay & $\mathrm{N}$ & $\mathrm{F}$ \\
\hline Echium vulgare L. & Boraginaceae & Viborera & I & $\mathrm{T}$ \\
\hline Embothrium coccineum J. R. Forst. et G. Forst. & Proteaceae & Notro & $\mathrm{N}$ & $\mathrm{F}$ \\
\hline Empetrum rubrum Vahl. ex Willd. & Empetraceae & Murtilla & $\mathrm{N}$ & $\mathrm{C}$ \\
\hline Erodium cicutarium (L. f) L'Herit. ex Aiton & Geraniaceae & Alfilerillo & I & $\mathrm{T}$ \\
\hline Escallonia rubra (Ruiz et Pav.) Pers. & Escalloniaceae & Nipa & $\mathrm{N}$ & $\mathrm{F}$ \\
\hline Escallonia virgata (Ruiz et Pav.) Pers. & Escalloniaceae & Chapel & $\mathrm{N}$ & $\mathrm{F}$ \\
\hline Euphorbia collina Phil. & Euphorbiaceae & Pichoga & I & $\mathrm{C}$ \\
\hline Festuca gracillima Hook. f. & Poaceae & Coirón & $\mathrm{N}$ & $\mathrm{H}$ \\
\hline Festuca rubra L. & Poaceae & Coirón & I & $\mathrm{H}$ \\
\hline Geranium berteroanus Colla & Geraniaceae & - & $\mathrm{N}$ & $\mathrm{H}$ \\
\hline Holcus lanatus L. & Poaceae & Pasto dulce & I & $\mathrm{H}$ \\
\hline Hordeum jubatum L. & Poaceae & Cebadilla & I & $\mathrm{T}$ \\
\hline Hordeum murinum L. & Poaceae & Cebadilla & I & $\mathrm{T}$ \\
\hline Loasa bergii Hieron. & Loasaceae & Ortiga & $\mathrm{N}$ & $\mathrm{T}$ \\
\hline Lupinus polyphyllus Lindl. & Fabaceae & Chocho & I & $\mathrm{F}$ \\
\hline Luzula racemosa Desv. & Juncaceae & - & $\mathrm{N}$ & $\mathrm{H}$ \\
\hline Madia sativa Molina & Asteraceae & Madi & $\mathrm{N}$ & $\mathrm{T}$ \\
\hline Mulinum spinosum (Cav.) Pers. & Apiaceae & Neneo & $\mathrm{N}$ & $\mathrm{C}$ \\
\hline Mutisia decurrens Cav. & Asteraceae & Clavel del aire & $\mathrm{N}$ & $\mathrm{F}$ \\
\hline Mutisia spinosa Ruiz et Pav. & Asteraceae & Clavel del aire & $\mathrm{N}$ & $\mathrm{F}$ \\
\hline Nothofagus antarctica (G. Forst.) Oerst. & Nothofagaceae & Ñire, Ñirre & $\mathrm{N}$ & $\mathrm{F}$ \\
\hline Nothofagus pumilio (Poepp. et Endl.) Krasser & Nothofagaceae & Lenga & $\mathrm{N}$ & $\mathrm{F}$ \\
\hline Oenothera stricta Ledeb. ex Link. & Onagraceae & Diego de la noche & $\mathrm{N}$ & $\mathrm{T}$ \\
\hline Phacelia secunda J. F. Gmel. & Hydrophyllaceae & Cuncuna & $\mathrm{N}$ & $\mathrm{H}$ \\
\hline Plantago lanceolata L. & Plantaginaceae & Siete venas & I & $\mathrm{H}$ \\
\hline Poa annua $\mathrm{L}$. & Poaceae & Pasto piojillo & I & $\mathrm{T}$ \\
\hline Ribes trilobum Meyen & Grossulariaceae & Zarzaparrilla & $\mathrm{N}$ & $\mathrm{F}$ \\
\hline Rumex acetosella $\mathrm{L}$. & Polygonaceae & Romacilla & I & $\mathrm{H}$ \\
\hline Schinus marchandii F.A. Barkley & Anacardiaceae & Laura & $\mathrm{N}$ & $\mathrm{F}$ \\
\hline Schinus patagonicus (Phil.) I.M. Johnst. & Anacardiaceae & Litrecillo & $\mathrm{N}$ & $\mathrm{F}$ \\
\hline Senecio darwinii Hook. et Arn. & Asteraceae & - & $\mathrm{N}$ & $\mathrm{C}$ \\
\hline Senecio patagonicus Hook. et Arn. & Asteraceae & - & $\mathrm{N}$ & $\mathrm{C}$ \\
\hline Solenomelus segethii (Phil.) Kuntze & Iridaceae & - & $\mathrm{N}$ & $\mathrm{Cr}$ \\
\hline Solidago chilensis Meyen & Asteraceae & Huelen & $\mathrm{N}$ & $\mathrm{Cr}$ \\
\hline Stipa sp. & Poaceae & Coirón & $\mathrm{N}$ & $\mathrm{H}$ \\
\hline Stipa speciosa Trin. et Rupr. & Poaceae & Coirón & $\mathrm{N}$ & $\mathrm{H}$ \\
\hline Taraxacum officinale G.Weber ex F. H. Wigg. & Asteraceae & Diente de león & $\mathrm{I}$ & $\mathrm{H}$ \\
\hline Tetraglochin alatum (Gillies ex Hook. et Arn.) Kuntze & Rosaceae & - & $\mathrm{N}$ & $\mathrm{F}$ \\
\hline Trifolium dubium Sibth. & Fabaceae & Trébol enano & I & $\mathrm{T}$ \\
\hline
\end{tabular}




\begin{tabular}{|c|c|c|c|c|}
\hline Nombre CIENTífico & FAMILIA & NOMBRE COMÚN & OR. & FV \\
\hline Trifolium pratense $\mathrm{L}$. & Fabaceae & Trébol rosado & I & $\mathrm{H}$ \\
\hline Trifolium repens $\mathrm{L}$. & Fabaceae & Trébol blanco & I & $\mathrm{H}$ \\
\hline Triptilium spinosum Ruiz et Pav. & Asteraceae & Siempreviva & $\mathrm{N}$ & $\mathrm{C}$ \\
\hline Valeriana carnosa $\mathrm{Sm}$. & Valerianaceae & - & $\mathrm{N}$ & $\mathrm{H}$ \\
\hline Verbascum thapsus L. & Scrophulariaceae & Hierba del paño & I & $\mathrm{T}$ \\
\hline Viola reichei Skottsb. & Violaceae & Violeta cimarrona & $\mathrm{N}$ & $\mathrm{H}$ \\
\hline Vulpia bromoides (L.) Gray & Poaceae & Vulpia & I & $\mathrm{T}$ \\
\hline
\end{tabular}

ORDENACIÓN TRADICIONAL DE LA TABLA INICIAL

Analizando la distribución de las presencias intermedias $(<11$ de frecuencia) en la tabla inicial fue posible distinguir 16 especies diferenciales, de las cuales 13 (Schinus patagonicus, Dactylis glomerata, Holcus lanatus, Mutisia spinosa, Trifolium repens, Acaena integerrima, Achillea millefolium, Baccharis obovata, Erodium cicutarium, Mutisia decurrens, Rumex acetosella, Anemone multifida y Baccharis magellanica) separan un grupo de vegetación y las otras tres (Schinus marchandii, Colliguaja integerrima y Solenomelus segetii) otro grupo (Tabla IV), ambos claramente distinguibles. Importante es señalar que en el primer grupo sólo 7 especies de las diferenciales son nativas y las otras 6 , introducidas, mientras que las 3 especies diferenciales del segundo grupo son nativas. Esto indica claramente que el primer grupo podría corresponder a una comunidad vegetal secundaria de reemplazo, mientras que la segunda, sería primaria, es decir, original y formada en el lugar.

ORDENACIÓN MULTIVARIADA DE LA TABLA INICIAL

La Fig. 3 muestra el dendrograma obtenido al analizar la tabla inicial, donde se forman 3 grupos en lugar de los dos encontrados ordenando la tabla por la metodología tradicional. Los dos primeros conglomerados (Fig. 3 A y B) se corresponden perfectamente con los grupos descritos en forma tradicional, pero el último está formado por una mezcla de ambos distribuidos en 2 subgrupos (Fig. 3 A'y $\mathrm{B}^{\prime}$ ), con los censos 4, 7 y 8 uno de ellos y con los censos 13, 15 y 18, el otro, correspondiendo cada uno de estos subgrupos a cada uno de los grupos descritos en forma tradicional. Al observar la tabla ordenada (Tabla IV) se aprecia que fue Mulinum spinosum, especie que no actúa como diferencial pero con alta frecuencia y cobertura, la que separó este tercer grupo pero actuando sola, es decir, con menos especies diferenciales que las usadas para la ordenación tradicional. Al utilizar un análisis de ordenación con la distribución de los censos en los dos primeros ejes, se observa que los censos 15,13 y 18 se integran claramente a los otros censos del segundo grupo determinado con la metodología tradicional, mientras que los del primer subgrupo se sitúan integrándose al primer grupo descrito, lo que valida la ordenación tradicional (Fig. 4). Además en esta figura se puede ver que los censos del primer grupo tradicional se encuentran más separados que los del segundo grupo, lo que habla de una mayor homogeneidad entre los censos del segundo grupo y una mayor heterogeneidad entre los censos del primero. Esta distribución espacial podría estar indicando que efectivamente el primer grupo descrito en forma tradicional es un grupo heterogéneo de origen secundario, que reemplaza algunas comunidades vegetales originales destruidas por el ser humano. Los diferentes estadios de degradación de esta comunidad secundaria podrían explicar su heterogeneidad florística.

\section{DESCRIPCIÓN DE LAS COMUNIDADES DETERMINADAS}

Una vez validados los grupos encontrados se describieron como asociaciones nuevas para Chile, ya que no se encuentran descritos en la literatura de vegetación de Chile ni Argentina.

ASOCIACIÓN: Mutisio-Schinetum patagonicae asoc. nova Comunidad de Mutisia spinosa y Schinus patagonicus Matorral de clavel del aire y molle

Se propone el censo $\mathrm{N}^{\circ} 2$ de la Tabla $\mathrm{V}$ como censo tipo. Se trata de una asociación arbustiva de origen secundario que reemplaza al bosque de lenga cuando es destruido, por lo tanto se la encuentra en la parte media del territorio de Aisén, cubierta por bosque caducifolio de lenga y matorrales de ñire. Los censos fueron levantados entre Villa Cerro Castillo y Cajón Cofré en la Carretera Austral. La tabla de esta comunidad (Tabla V) presenta 55 especies de las cuales 31 son nativas y 24 introducidas, esta alta cantidad de especies alóctonas la caracteriza como una comunidad secundaria. El promedio de especies por censo alcanzó a 17,6. El valor de importancia más alto $(31,2)$ de la tabla lo presenta Schinus patagonicus que también tiene la mayor cobertura absoluta (370\%). Siguen con menores valores de importancia Mulinum spinosum $(15,3)$ y Acaena pinnatifida $(14,8)$ ambas nativas, la primera es un subarbusto indicador de suelos degradados y la segunda es una típica especie ruderal indicadora de intervención humana. 
ASOCIACIÓN: Colliguajo-Schinetum marchandii asoc. nova Comunidad de Colliguaja integerrima y Schinus marchandii Estepa arbustiva de duraznillo y laura.

Se propone el censo $\mathrm{N}^{\circ} 15$ de la Tabla VI que la representa, como censo tipo. Corresponde a una asociación arbustiva primaria con carácter estepario. Los censos de vegetación fueron levantados en los alrededores de Chile Chico en la ruta de esta ciudad a la Reserva Nacional "Lago Jeinimeni". La tabla de vegetación presenta 24 especies, de las cuales sólo dos son alóctonas, Verbascum thapsus y Hordeum jubatum, lo que confirma su carácter de asociación primaria original del lugar. El promedio de especies por censo alcanzó a 8,9. Colliguaja integerrima fue la especie más importante, con un valor de importancia 54,2 y también la dominante con una cobertura total de $355 \%$, ésta sirvió como diferencial. Le siguen Mulinum spinosum con un valor de importancia de 26,3 y una cobertura total de 152, Berberis microphylla con 23 de valor de importancia y $122 \%$ de cobertura y en cuarto lugar aparece Schinus marchandii con un valor de importancia de 20,9 y una cobertura total de sólo 7,8\%, pero es la única especie que estuvo presente en todos los censos, es decir, con 100 de frecuencia. La primera es una planta pulvinada propia de estepas y la segunda, un arbusto muy abundante y presente en varias comunidades vegetales.

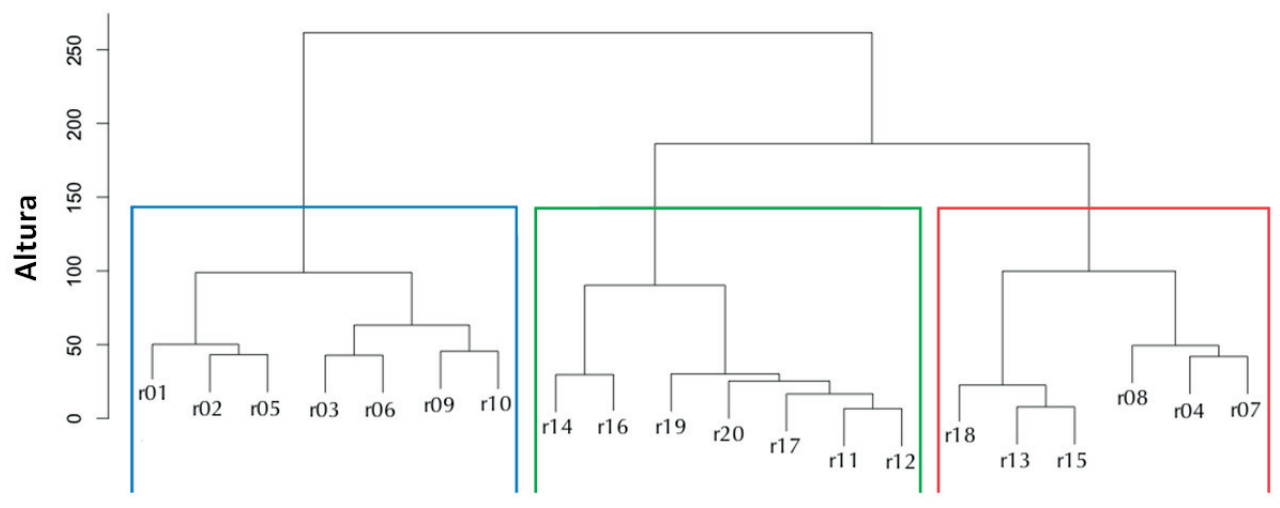

Figura 3. Dendrograma que clasifica los censos de muestreo en tres grupos según la distancia florística de Bray-Curtis entre ellos. Los números antecedidos de una letra " $r$ " corresponden a los relevamientos o censos de vegetación de la tabla inicial (Tabla 4). Mayores explicaciones en el texto.

FiguRE 3. Dendrogram which classifies de vegetación samples into three groups according to the floristic Bray-Curtis distance between them. The numbers preceded by the letter " $r$ " correspond to the relevés or vegetación samples of the initial plant sociological table (Table 4). Further explanation in the text.

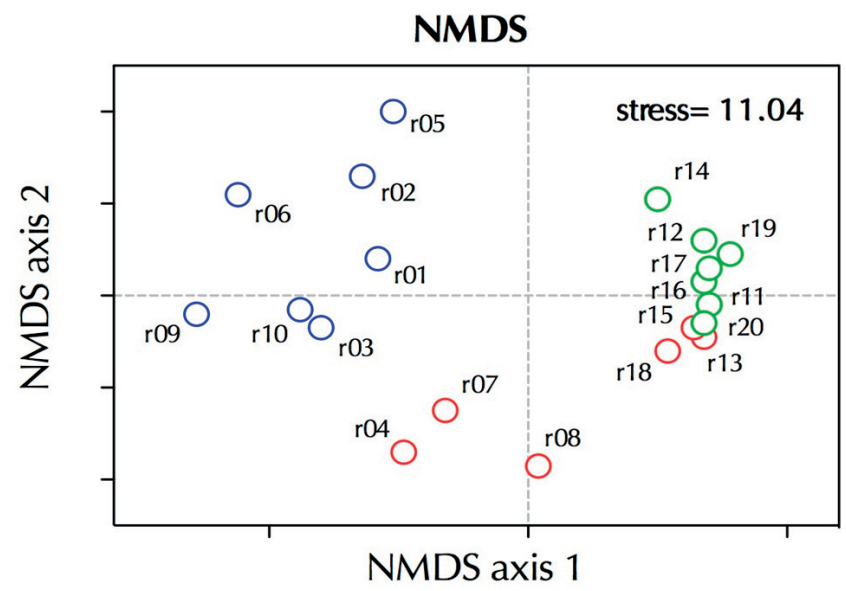

Figura 4. Ordenación de los censos fitosociológicos en los dos primeros ejes del Análisis Multidimensional no-métrico (NMDS), con un valor de estrés aceptable.

FIGURE 4. Management of plant sociological samples in the first two axes of the Non-Metric Multidimensional Analysis (NMDS), with an acceptable stress value. 
Hábitats de especies de Schinus en Aisén: Ramírez, C. ET AL.

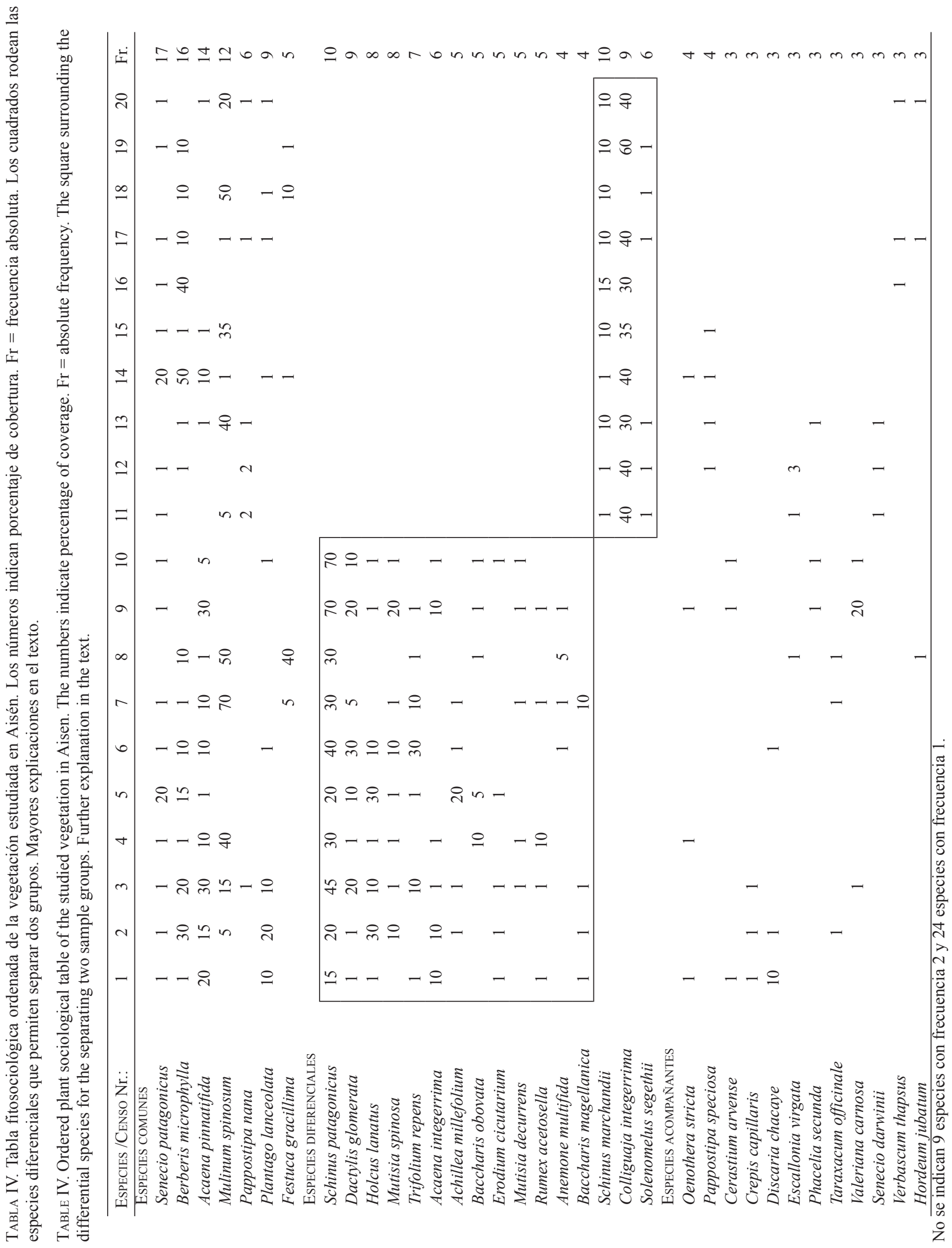


TABLA V. Estructura fitosociológica de asociación Mutisio-Schinetum patagonicae. Fr. $=$ frecuencia, Fr.rel = frecuencia relativa, Cob.abs $=$ cobertura absoluta, Cob.rel $=$ cobertura relativa, V.I. $=$ valor de importancia. Censo tipo $=2$.

TABLE V. Floristic structure of the new plant association Mutisio-Schinetum patagonicae. Fr $=$ frequency, Fr.rel $=$ relative frequency, Cob. abs $=$ absolute coverage, Cob.rel $=$ relative coverage, I.V. $=$ importance value. Type vegetation sample $=2$.

\begin{tabular}{|c|c|c|c|c|c|c|c|c|c|c|c|c|c|c|c|}
\hline Especies / Censo Nr.: & 1 & 2 & 3 & 4 & 5 & 6 & 7 & 8 & 9 & 10 & Fr. & FR.REL & CoB.ABs & CoB.REL & V.I. \\
\hline Schinus patagonicus & 15 & 20 & 45 & 30 & 20 & 40 & 30 & 30 & 70 & 70 & 10 & 5,7 & 370 & 25,6 & 31,2 \\
\hline Acaena pinnatifida & 20 & 15 & 30 & 10 & 1 & 10 & 10 & 1 & 30 & 5 & 10 & 5,7 & 132 & 9,1 & 14,8 \\
\hline Dactylis glomerata & 1 & 1 & 20 & 1 & 10 & 30 & 5 & & 20 & 10 & 9 & 5,1 & 98 & 6,8 & 11,9 \\
\hline Senecio patagonicus & 1 & 1 & 1 & 1 & 20 & 1 & 1 & & 1 & 1 & 9 & 5,1 & 28 & 1,9 & 7,0 \\
\hline Berberis microphylla & 1 & 30 & 20 & 1 & 15 & 10 & 1 & 10 & & & 8 & 4,5 & 88 & 6,1 & 10,6 \\
\hline Holcus lanatus & 1 & 30 & 10 & 1 & 30 & 10 & & & 1 & 1 & 8 & 4,5 & 84 & 5,8 & 10,3 \\
\hline Mutisia spinosa & & 10 & 1 & 1 & 1 & 10 & 1 & & 20 & 1 & 8 & 4,5 & 45 & 3,1 & 7,7 \\
\hline Trifolium repens & 1 & & 10 & & 1 & 30 & 10 & 1 & 1 & & 7 & 4,0 & 54 & 3,7 & 7,7 \\
\hline Acaena integerrima & 10 & 10 & 1 & 1 & & & & & 10 & 1 & 6 & 3,4 & 33 & 2,3 & 5,7 \\
\hline Mulinum spinosum & & 5 & 15 & 40 & & & 70 & 50 & & & 5 & 2,8 & 180 & 12,4 & 15,3 \\
\hline Plantago lanceolata & 10 & 20 & 10 & & & 1 & & & & 1 & 5 & 2,8 & 42 & 2,9 & 5,7 \\
\hline Achillea millefolium & & 1 & 1 & & 20 & 1 & 1 & & & & 5 & 2,8 & 24 & 1,7 & 4,5 \\
\hline Baccharis obovata & & & & 10 & 5 & & & 1 & 1 & 1 & 5 & 2,8 & 18 & 1,2 & 4,1 \\
\hline Rumex acetosella & 1 & & 1 & 10 & & & 1 & & 1 & & 5 & 2,8 & 14 & 1,0 & 3,8 \\
\hline Erodium cicutarium & 1 & 1 & 1 & & 1 & & & & & 1 & 5 & 2,8 & 5 & 0,3 & 3,2 \\
\hline Mutisia decurrens & & & 1 & 1 & & & 1 & & 1 & 1 & 5 & 2,8 & 5 & 0,3 & 3,2 \\
\hline Baccharis magellanica & 1 & 1 & 1 & & & & 10 & & & & 4 & 2,3 & 13 & 0,9 & 3,2 \\
\hline Anemone multifida & & & & & & 1 & 1 & 5 & 1 & & 4 & 2,3 & 8 & 0,6 & 2,8 \\
\hline Valeriana carnosa & & & 1 & & & & & & 20 & 1 & 3 & 1,7 & 22 & 1,5 & 3,2 \\
\hline Discaria chacaye & 10 & 1 & & & & 1 & & & & & 3 & 1,7 & 12 & 0,8 & 2,5 \\
\hline Cerastium arvense & 1 & & & & & & & & 1 & 1 & 3 & 1,7 & 3 & 0,2 & 1,9 \\
\hline Crepis capillaris & 1 & 1 & 1 & & & & & & & & 3 & 1,7 & 3 & 0,2 & 1,9 \\
\hline Oenothera stricta & 1 & & & 1 & & & & & 1 & & 3 & 1,7 & 3 & 0,2 & 1,9 \\
\hline Taraxacum officinale & & 1 & & & & & 1 & 1 & & & 3 & 1,7 & 3 & 0,2 & 1,9 \\
\hline Festuca gracillima & & & & & & & 5 & 40 & & & 2 & 1,1 & 45 & 3,1 & 4,2 \\
\hline Colletia hystrix & & & & 20 & & & & & & 20 & 2 & 1,1 & 40 & 2,8 & 3,9 \\
\hline Nothofagus antarctica & & & & & & 20 & & & & 10 & 2 & 1,1 & 30 & 2,1 & 3,2 \\
\hline Vulpia bromoides & & & & 5 & & & & & & 1 & 2 & 1,1 & 6 & 0,4 & 1,6 \\
\hline Geranium berteroanus & & 1 & & & & 1 & & & & & 2 & 1,1 & 2 & 0,1 & 1,3 \\
\hline Hordeum murinum & 1 & & & 1 & & & & & & & 2 & 1,1 & 2 & 0,1 & 1,3 \\
\hline Madia sativa & & & & & 1 & 1 & & & & & 2 & 1,1 & 2 & 0,1 & 1,3 \\
\hline Phacelia secunda & & & & & & & & & 1 & 1 & 2 & 1,1 & 2 & 0,1 & 1,3 \\
\hline Viola reichei & & & & & & 1 & & & 1 & & 2 & 1,1 & 2 & 0,1 & 1,3 \\
\hline Embothrium coccineum & & & & & & & & & & 5 & 1 & 0,6 & 5 & 0,3 & 0,9 \\
\hline Nothofagus pumilio & 5 & & & & & & & & & & 1 & 0,6 & 5 & 0,3 & 0,9 \\
\hline Agrostis capillaris & & & & & 1 & & & & & & 1 & 0,6 & 1 & 0,1 & 0,6 \\
\hline Aira caryophyllea & & & & & & & & 1 & & & 1 & 0,6 & 1 & 0,1 & 0,6 \\
\hline Amaranthus deflexus & & & & 1 & & & & & & & 1 & 0,6 & 1 & 0,1 & 0,6 \\
\hline Bromus catharticus & & & & & & & 1 & & & & 1 & 0,6 & 1 & 0,1 & 0,6 \\
\hline Chamomilla suaveolens & 1 & & & & & & & & & & 1 & 0,6 & 1 & 0,1 & 0,6 \\
\hline Cirsium vulgare & & & & & 1 & & & & & & 1 & 0,6 & 1 & 0,1 & 0,6 \\
\hline Echium vulgare & & 1 & & & & & & & & & 1 & 0,6 & 1 & 0,1 & 0,6 \\
\hline Escallonia rubra & & & & 1 & & & & & & & 1 & 0,6 & 1 & 0,1 & 0,6 \\
\hline
\end{tabular}




\begin{tabular}{|c|c|c|c|c|c|c|c|c|c|c|c|c|c|c|c|}
\hline Especies / Censo NR.: & 1 & 2 & 3 & 4 & 5 & 6 & 7 & 8 & 9 & 10 & Fr. & FR.REL & CoB.ABS & COB.REL & V.I. \\
\hline Escallonia virgata & & & & & & & & 1 & & & 1 & 0,6 & 1 & 0,1 & 0,6 \\
\hline Festuca rubra & & & & & & & 1 & & & & 1 & 0,6 & 1 & 0,1 & 0,6 \\
\hline Hordeum jubatum & & & & & & & & 1 & & & 1 & 0,6 & 1 & 0,1 & 0,6 \\
\hline Stipa sp. & & & 1 & & & & & & & & 1 & 0,6 & 1 & 0,1 & 0,6 \\
\hline Loasa bergii & & & 1 & & & & & & & & 1 & 0,6 & 1 & 0,1 & 0,6 \\
\hline Lupinus polyphyllus & & & & 1 & & & & & & & 1 & 0,6 & 1 & 0,1 & 0,6 \\
\hline Luzula racemosa & & & & & & & 1 & & & & 1 & 0,6 & 1 & 0,1 & 0,6 \\
\hline Poа аппиа & 1 & & & & & & & & & & 1 & 0,6 & 1 & 0,1 & 0,6 \\
\hline Ribes trilobum & & & & & & & & 1 & & & 1 & 0,6 & 1 & 0,1 & 0,6 \\
\hline Trifolium dubium & & & & & & & & & 1 & & 1 & 0,6 & 1 & 0,1 & 0,6 \\
\hline Trifolium pratense & & & 1 & & & & & & & & 1 & 0,6 & 1 & 0,1 & 0,6 \\
\hline Triptilium spinosum & 1 & & & & & & & & & & 1 & 0,6 & 1 & 0,1 & 0,6 \\
\hline Total especies (55) & 21 & 18 & 21 & 19 & 14 & 16 & 18 & 13 & 18 & 18 & 176 & 100 & 1448 & 100 & 200 \\
\hline
\end{tabular}

Los números de los censos indican porcentaje de cobertura./ Census numbers indicate the percentage of cover.

TABLA VI. Estructura fitosociológica de asociación Colliguajo-Schinetum marchandii. Fr. = frecuencia, Fr.rel = frecuencia relativa, Cob. abs $=$ cobertura absoluta, Cob.rel $=$ cobertura relativa, V.I. $=$ valor de importancia. Censo tipo $=15$.

TABLA VI. Floristic structure of the new association Colliguajo-Schinetum marchandii. $\mathrm{Fr}=$ frequency, Fr.rel = relative frequency, Cob. abs $=$ absolute coverage, Cob.rel $=$ relative coverage, I.V. $=$ importance value. Type vegetation sample $=15$.

\begin{tabular}{|c|c|c|c|c|c|c|c|c|c|c|c|c|c|c|c|}
\hline ESPECIES / CENSO Nr.: & 11 & 12 & 13 & 14 & 15 & 16 & 17 & 18 & 19 & 20 & $\begin{array}{c}\text { F. } \\
\text { ABS }\end{array}$ & $\begin{array}{c}\text { F. } \\
\text { REL }\end{array}$ & $\begin{array}{l}\text { Coв. } \\
\text { ABS }\end{array}$ & $\begin{array}{c}\text { Cob. } \\
\text { ReL }\end{array}$ & V.I. \\
\hline Schinus marchandii & 1 & 1 & 10 & 1 & 10 & 15 & 10 & 10 & 10 & 10 & 10 & 11,2 & 78 & 9,7 & 20,9 \\
\hline Colliguaja integerrima & 40 & 40 & 30 & 40 & 35 & 30 & 40 & & 60 & 40 & 9 & 10,1 & 355 & 44,0 & 54,2 \\
\hline Senecio patagonicus & 1 & 1 & & 20 & 1 & 1 & 1 & & 1 & 1 & 8 & 9,0 & 27 & 3,3 & 12,3 \\
\hline Mulinum spinosum & 5 & & 40 & 1 & 35 & & 1 & 50 & & 20 & 7 & 7,9 & 152 & 18,9 & 26,7 \\
\hline Berberis microphylla & & 1 & & 50 & 1 & 40 & 10 & 10 & 10 & & 7 & 7,9 & 122 & 15,1 & 23,0 \\
\hline Solenomelus segethii & 1 & 1 & 1 & & & & 1 & 1 & 1 & & 6 & 6,7 & 6 & 0,7 & 7,5 \\
\hline Stipa sp. & 2 & 2 & 1 & & & & 1 & & & 1 & 5 & 5,6 & 7 & 0,9 & 6,5 \\
\hline Acaena pinnatifida & & & 1 & 10 & 1 & & & & & 1 & 4 & 4,5 & 13 & 1,6 & 6,1 \\
\hline Stipa speciosa & & 1 & 1 & 1 & 1 & & & & & & 4 & 4,5 & 4 & 0,5 & 5,0 \\
\hline Plantago lanceolata & & & & 1 & & & 1 & 1 & & 1 & 4 & 4,5 & 4 & 0,5 & 5,0 \\
\hline Festuca gracillima & & & & 1 & & & & 10 & 1 & & 3 & 3,4 & 12 & 1,5 & 4,9 \\
\hline Senecio darwinii & 1 & 1 & 1 & & & & & & & & 3 & 3,4 & 3 & 0,4 & 3,7 \\
\hline Verbascum thapsus & & & & & & 1 & 1 & & & 1 & 3 & 3,4 & 3 & 0,4 & 3,7 \\
\hline Escallonia virgata & 1 & 3 & & & & & & & & & 2 & 2,2 & 4 & 0,5 & 2,7 \\
\hline Adesmia boronoides & & 1 & & & 1 & & & & & & 2 & 2,2 & 2 & 0,2 & 2,5 \\
\hline Euphorbia collina & 1 & & 1 & & & & & & & & 2 & 2,2 & 2 & 0,2 & 2,5 \\
\hline Hordeum jubatum & & & & & & & 1 & & & 1 & 2 & 2,2 & 2 & 0,2 & 2,5 \\
\hline Oenothera stricta & 1 & & & 1 & & & & & & & 2 & 2,2 & 2 & 0,2 & 2,5 \\
\hline Tetraglochin alatum & 3 & & & & & & & & & & 1 & 1,1 & 3 & 0,4 & 1,5 \\
\hline Acaena tenera & & & 1 & & & & & & & & 1 & 1,1 & 1 & 0,1 & 1,2 \\
\hline Adesmia lotoides & & & & 1 & & & & & & & 1 & 1,1 & 1 & 0,1 & 1,2 \\
\hline Empetrum rubrum & & & & & 1 & & & & & & 1 & 1,1 & 1 & 0,1 & 1,2 \\
\hline Phacelia secunda & & & 1 & & & & & & & & 1 & 1,1 & 1 & 0,1 & 1,2 \\
\hline Solidago chilensis & & & & & & & 1 & & & & 1 & 1,1 & 1 & 0,1 & 1,2 \\
\hline Total especies (24): & 11 & 10 & 11 & 11 & 9 & 5 & 11 & 6 & 6 & 9 & 89 & 100 & 806 & 100 & 200 \\
\hline
\end{tabular}

Los números de los 10 censos indican porcentaje de cobertura./ Census numbers indicate the percentage of cover. 


\section{COMPARACIÓN ENTRE LAS ASOCIACIONES}

De las 68 especies que conforman la tabla inicial 11 son comunes a las dos asociaciones, 13 son exclusivas del matorral estepario del Colliguajo-Schinetum marchandii y 44, exclusivas del matorral secundario del MutisioSchinetum patagonicae. De las especies comunes nueve son nativas (Acaena pinnatifida, Berberis microphylla, Escallonia virgata, Festuca gracillima, Stipa sp., Mulinum spinosum, Oenothera stricta, Senecio patagonicus y Phacelia secunda, mientras que las dos restantes (Hordeum jubatum y Plantago lanceolata) son introducidas.

Al calcular el índice de Jaccard la afinidad florística entre las dos asociaciones es de un 16\%, es decir tienen escasa similitud siendo dos comunidades bien definidas. Con el coeficiente de asociación de Ellenberg calculado con la cobertura promedio esta similitud florística sube a $24,5 \%$, un valor muy cercano a este $(23,5 \%)$ se presenta al hacer el cálculo ponderando la cobertura. Estos resultados confirman que las comunidades que conforman el hábitat de cada una de las dos especies Schinus patagonicus y Schinus marchandii en la Región de Aisén son totalmente diferentes

Al comparar los espectros biológicos de ambas comunidades se observa que son también diferentes ya que el porcentaje de fanerófitos, hemicriptófitos y de terófitos es más alto en el Mutisio-Schinetum patagonicae y baja en la estepa del Colliguajo-Schinetum marchandii, mientras que los caméfitos y los criptófitos suben en el último matorral, incluso esta última forma de vida no está presente en el primero de ellos (Tabla VII). El comportamiento del número de estas dos últimas formas de vida señala que el microclima del hábitat de Schinus marchandii es más extremo, con una mayor sequía, temperatura y velocidad del viento, es decir de un macroclima estepario. Sin embargo, la presencia de abundantes terófitos debería ser propia de semejante macroclima, pero por el contrario

TABLA VII. Espectro biológico de la flora acompañante de los Schinus de Aisén.

TABLE VII. Biological spectrum of the accompanying flora of Schinus Aisen.

\begin{tabular}{lcccc}
\hline AsOCIACIÓN & \multicolumn{2}{c}{ Mutisio-Schinetum } & patagonicae & \multicolumn{2}{c}{ Colliguajo-Schinetum marchandii } \\
\hline Forma de vida & Especies & Porcentaje & Especies & Porcentaje \\
\hline Fanerófitos & 14 & 25,45 & 5 & 20,83 \\
Caméfitos & 6 & 10,91 & 7 & 29,17 \\
Hemicriptófitos & 19 & 34,55 & 7 & 29,17 \\
Criptófitos & 0 & 0 & 2 & 8,33 \\
Terófitos & 16 & 29,09 & 3 & 12,50 \\
\hline Total & 55 & 100 & 24 & 100 \\
\hline
\end{tabular}

Tabla VIII. Diferenciación de los hábitats de Schinus patagonicus y Schinus marchandii en Aisén, Chile.

TABLE VIII. Habitats differentiation of Schinus patagonicus and Schinus marchandii in Aisen, Chile.

\begin{tabular}{lcc}
\hline CARACTERÍsTiCAS / ESPECIE & Schinus patagonicus & Schinus marchandii \\
\hline Asociación vegetal & Mutisio-Schinetum patagonicae & Colliguajo-Schinetum marchandii \\
Comunidad & Matorral de clavel del aire y molle & Matorral de duraznillo y laura \\
Zona de vegetación & Continental de bosques caducifolios & Esteparia arbustiva \\
Origen & Secundaria & Primaria \\
Comunidad que reemplaza & Bosque de lenga y matorral de ñire & Ninguna \\
Sustrato & Rocoso & Arenoso \\
Límites altitudinales & $320-470$ m s.n.m. & $205-633$ m s.n.m. \\
Zona de mayor abundancia & De Villa Cerro Castillo a Cajón Cofre & En alrededores de Chile Chico \\
Clima & Húmedo y frío de bosques caducifolios & Seco y caluroso de estepa \\
Especies en la tabla fitosociológica & 55 & 24 \\
Especies nativas & 31 & 22 \\
Especies introducidas & 24 & 2 \\
Cobertura promedio & $37 \%$ & $7,8 \%$ \\
\hline
\end{tabular}



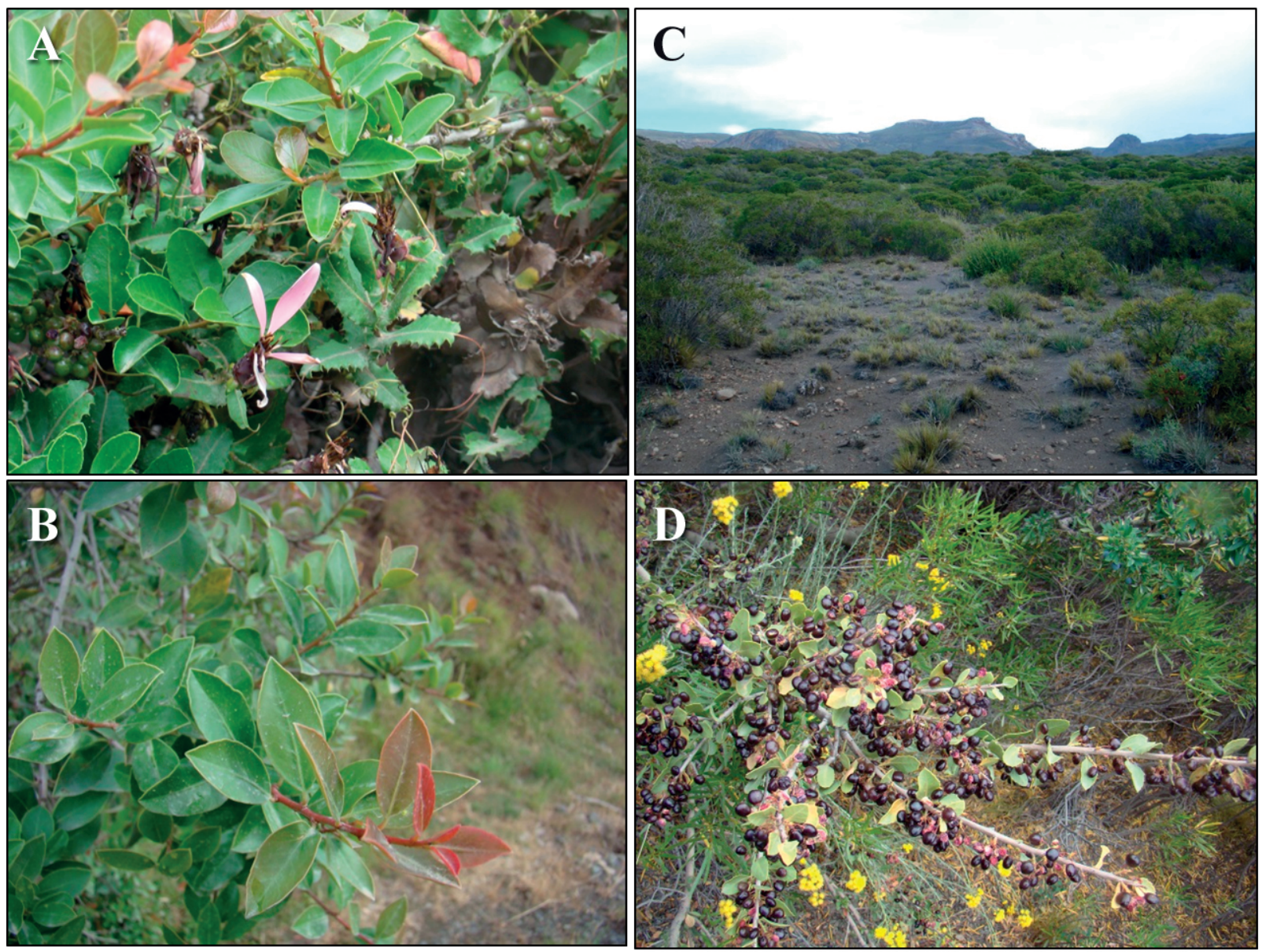

Figura 5. Aspecto de la asociación Mutisio-Schinetum patagonicae (A), detalle de Schinus patagonicus (B), aspecto del ColliguajoSchinetum marchandii (C) y detalle de Schinus marchandii (D).

FIGURE 5. Appearance of Mutisio-Schinetum patagonicae association (A), detail of Schinus patagonicus (B) aspect of ColliguajoSchinetum marchandii (C) and detail of Schinus marchandii (D).

esta forma de vida se presenta con un valor muy alto en el matorral del Mutisio-Schinetum patagonicae con un macroclima más húmedo y frío. Lo anterior tiene su explicación en la gran cantidad de malezas presentes en esta última asociación que son plantas anuales, propias de hábitats con características xerofíticas por la mayor sequía que provoca la compactación del suelo causada por el pisoteo. De acuerdo a lo anterior los hábitats de las especies Schinus patagonicus y Schinus marchandii caracterizados por la presencia de asociaciones vegetales diferentes, se pueden comparar en la Tabla VIII. Estas diferencias se pueden apreciar también en la Fig. 5.

\section{DISCUSIÓN}

Las diferencias de hábitats encontradas para Schinus patagonicus y Schinus marchandii son muy claras, el primero crece en matorrales secundarios de la zona intermedia donde primitivamente existían formaciones vegetales caducifolias principalmente de Nothofagus pumilio y el segundo en matorrales xerófitos primarios de la estepa patagónica oriental, en el límite con Argentina. En el primer caso la acción antrópica es evidente ya que es causante de la degradación de la vegetación primitiva caducifolia y de la formación de los matorrales secundarios que sirven de hábitats a Schinus patagonicus, que prospera muy bien reproduciéndose en forma exitosa y dominando en ellos (Roig 1994). Por el contrario, en el segundo caso la acción humana aunque está presente por la mejor accesibilidad, no es tan evidente, aunque si se toma en cuenta los relatos de lugareños, Schinus marchandii se presentaba antiguamente como arbolito de hasta $6 \mathrm{~m}$ de alto, ejemplares que hoy en día no existen, ya que fueron utilizados como leña principalmente, además, esta especie no es dominante en su comunidad, aunque siempre está presente en ella (Roig 1994, Saldivia \& Rojas 2008). La acción antrópica se deja ver también en el ramoneo del ganado. 
Una asociación vegetal próxima al Mutisio-Schinetum patagonicae ha sido descrita por Eskuche (1969) con el nombre de Mutisio-Berberidietum darwinii, pero en la primera asociación faltan especies importantes y diferenciales para esta última, como son Berberis darwinii, Maytenus boaria, Nothofagus dombeyi, Austrocedrus chilensis y Lomatia hirsuta. A su vez, en esta última comunidad faltan especies muy importantes y diferenciales del MutisioSchinetum patagonicae, como son Acaena pinnatifida y Acaena integerrima, Mulinum spinosum, Dactylis glomerata y Senecio patagonicus, entre otros. Al parecer la asociación de Eskuche sería un matorral secundario restringido al lado argentino, donde reemplazaría a los bosques LomatioNothofagetum dombeyii y Austrocedro-Nothofagetum dombeyii, que son bosques perennifolios, dominados por el coihue (Nothofagus dombeyi), incluso presenta una variante con Chusquea culeou, especie de bambú que tampoco está presente en el Mutisio-Schinetum patagonicae, pero que si es abundante en el sotobosque de bosques riparios de coihue en el lado chileno (Chusqueo-Nothofagetum dombeyii) (Vidal et al. 2011), cuyos matorrales secundarios carecen de Schinus patagonicus. La segunda asociación vegetal aquí descrita Colliguajo-Schinetum marchandii, ha sido citada por Roig (1994), sin llegar a nombrarla, pero estableciendo que la especie dominante en ella, Schinus marchandii, se presenta como una forma arbustiva, indicando degradación de esa comunidad esteparia, lo que también se plantea en este trabajo. Esto último está de acuerdo con la abundante presencia de Mulinum spinosum en ambos matorrales que podría estar indicando degradación antrópica de la vegetación, ya que esta especie tiende a ocupar espacios denudados artificialmente, especialmente aquellos con vegetación primaria boscosa y arbustiva (Vidal 2007, San Martín et al. 2009, Ramírez et al. 2012). La presencia abundante de esta especie que permitió la segregación de subgrupos en ambas comunidades, aun cuando no es una especie diferencial por su baja fidelidad fitosociológica hacia ellas, siendo más bien una especie característica de las estepas aiseninas, podría estar indicando intervención antrópica que es evidente en el Mutisio-Schinetum patagonicae, pero no parece tan claro en el matorral estepario de Colliguajo-Schinetum marchandii, porque en la estepa esta especie aparece formando parte de algunas comunidades vegetales primarias. La alteración de la vegetación en la Región de Aisén es evidente y ha sido mencionada por Ramírez et al. (2012) y especialmente, por la gran cantidad de malezas alóctonas integradas a las comunidades, incluso como especies diferenciales, pero aún faltan estudios sobre las sucesiones secundarias para aclarar la dinámica de degradación y permitir la protección o un manejo sustentable de los hábitats, especialmente faltan aproximaciones a la sintaxonomía de ellas, que para la primera comunidad descrita podría corresponder al Orden Berberidion buxifoliae perteneciente a la Clase
Wintero-Nothofagetea de Oberdorfer (1960). Estudios de degradación de la vegetación de la parte norte de la Patagonia chilena han sido realizados por Hildebrand-Vogel (1988) en comunidades nativas boscosas siempre verdes.

Aunque aún hay problemas nomenclaturales y de delimitación de las especies de Schinus en Chile, se sabe que las dos especies de Aisén están presentes más al norte, pero no en forma abundante, esto es especialmente válido para Schinus marchandii, que además de ocupar un hábitat extremo en la Región de Aisén, sólo ha sido recolectado y citado esporádicamente entre las regiones de la Araucanía recolectado por Saavedra en 1993 (SGO 133704) y de Los Ríos (Ramírez et al. 1990). Este último ejemplar desapareció posteriormente (Lagos et al. 2000). Por todo lo anterior, esta última especie debería ser protegida en Chile como lo solicita la CONAF (2012), aun cuando está ampliamente distribuida en Argentina. Un factor importante y que indica la precariedad en que viven estas especies lo constituye una fuerte disminución de la superficie foliar en ambas por causa desconocida. En Schinus patagonicus se observó muerte de ramas y hojas que permanecen en la planta y en Schinus marchandii una notoria defoliación por caída (o por no formación) de hojas.

Los análisis multivariados de la tabla fitosociológica inicial permiten no sólo validar los grupos segregados por métodos tradicionales, sino que también permiten formarse una idea de la dinámica de la degradación antrópica y de la importancia de cada especie en las diferentes comunidades involucradas.

El estudio y descripción de estas dos nuevas asociaciones vegetales permite conocer y diferenciar los hábitats en que crecen ambas especies, facilitando así acciones de conservación de ellas, mediante la protección de las comunidades que forman sus hábitats o lugares de vida, lo que es especialmente importante para Schinus marchandii, especie considerada actualmente con problemas de conservación en Chile.

\section{AGRADECIMIENTOS}

Trabajo financiado a través del Proyecto CONAF-UACh "Actualización del Catastro de Bosque Nativo Región de Aisén, Chile".

\section{BIBLIOGRAFÍA}

Álvarez, M., C. San Martín, C. Novoa, G. Toledo \& C. Ramírez. 2010. Diversidad florística, vegetacional y de hábitats en el Archipiélago de Los Chonos (Región de Aisén, Chile). Anales del Instituto de la Patagonia (Chile) 38(1): 34-55.

Amigo, J. \& C. Ramírez. 1998. A bioclimatic classification of Chile: woodland communities in the temperate zone. Plant Ecology 136: 9-26. 
Badil, M., J. Landeros \& E. Cerna. 2007. Patrones de asociación de especies y sustentabilidad. Daena: International Journal of Good Conscience 3(1): 632-660.

BARKLey, F.A. 1944. Schinus L. Brittonia 5(2): 160-198.

BARKLeY, F.A. 1957. A study of Schinus L. Lilloa 28: 5-109.

Braun-Blanquet, J. 1979. Fitosociología. Bases para el estudio de las comunidades vegetales. H. Blume Ediciones, Madrid. $820 \mathrm{pp}$.

CABrera, A.L. 1938. Revisión de las Anacardiáceas austroamericanas. Revista del Museo La Plata Sección Botánica 2: 3-64.

Clarke, K.R. 1993. A method of linking multivariate community structure to environmental variables. Marine Ecology Progress Series 92: 205-219.

Comunidades Europeas. 1992. Directiva Hábitat. Diario Oficial de las Comunidades Europeas L206/7.

CONAF. 2012. Ficha con una propuesta preliminar de clasificación de Schinus marchandii como en Peligro Crítico. Santiago. 4 pp.

Dengler, J., M. Chytry \& J. Ewald. 2008. Phytosociology. In: S.E. Jøgensen, \& B.D. Fath (eds.), Encyclopedia of Ecology. pp. 2767-2779. Elsevier, Oxford, England.

DierschKe, H. 1994. Pflanzensoziologie - Grundlagen und Methoden. Eugen Ulmer, Stuttgart. 683 pp.

EsKuCHE, U. 1969. Berberitzengebüsche und Nothofagus antarctica-Wälder in Nordwestpatagonien. Vegetatio 16(1-4): 192-204.

GAJARDO, R. 1994. La vegetación natural de Chile - Clasificación y Distribución geográfica. Editorial Universitaria, CONAF, Santiago. 165 pp.

Hajek, E. \& F. Di CAstri. 1975. Bioclimatografía de Chile. Editorial Universidad Católica de Chile, Santiago. 107 pp.

Hildebrand-Vogel, R. 1988. Ersatzgesellschaften des nordwestpatagonichen Lobeerwaldes in Chile zwischen 42 und $45^{\circ}$ s. Br. Flora 180: 161-176.

Isla, F., E. Ruiz, J. Márquez \& A. Urrutia. 2003. Efectos ENSO en la transición entre el espinal y la pradera cultivada en la Diagonal Sudamericana, Argentina Central. Revista Cuaternario \& Geología 17(1-2): 63-74.

IzCo, J. \& M. DEL ARCo. 2003. Código internacional de nomenclatura fitosociológica. Materiales Didácticos Universitarios. Universidad de Laguna, Serie Botánica 2: 9-154.

KNAPP, R. 1984. Considerations on qualitative parameters and qualitative attributes in vegetation analysis and in phytosociological relevés. In: R. Knapp (ed.), Sampling methods and taxon analysis in vegetation science, pp 77119. Dr. W. Junk Pub. La Haya.

Lagos, R., M. Cruz, C. Espinoza \& C. Ramírez. 2000. Fitogeografía de Peumus boldus Mol. en la hoya del río Bueno, Región de Los Lagos, Chile. Boletín de Geografía 12-13: 47-60.

Legendre P. \& L. Legendre. 1998. Numerical ecology. Elsevier, Amsterdam. 853 pp.

Luebert, F. \& P. Pliscoff. 2006. Sinopsis bioclimática y vegetacional de Chile. Editorial Universitaria, Santiago. 316 pp.

McCune, B. \& J. B. Grace. 2002. Analysis of ecological communities. MJM Software, Glenden Beach, Oregon, USA. 547 pp.

Ministerio del Medio Ambiente. 2013. Evaluando el estado de conservación de nuestras especies nativas y difundiendo el conocimiento sobre las especies que viven en Chile.
Santiago. $32 \mathrm{pp}$.

Mueller-Dombois, D. \& H. Ellenberg. 1974. Aims and methods of vegetation ecology. John Wiley \& Sons. New York. 547 pp.

Muñoz, C. 1966. Sinopsis de la flora chilena - claves para la identificación de familias y géneros. Ediciones de la Universidad de Chile, Santiago. 500 pp.

Novoa, R. \& S. Villaseca. 1989. Agroclimatología de Chile. Instituto de Investigaciones Agropecuarias (INIA), Ministerio de Agricultura, Santiago. 211 pp.

Oberdorfer, E. 1960. Pflanzensoziologischen Studien in Chile. Ein Vergleich mit Europa. Flora et Vegetatio mundi 2: $1-108$.

Quintanilla, V. 1989. Fitogeografía y cartografía vegetal de Chile austral. Contribuciones científicas y tecnológicas, Area Geociencias VII 21(87): 1-27,1 mapa.

R Development Core_Team. 2012. R: a language and environment for statistical computing. Vienna, Austria. http://www.Rproject.org

Ramírez, C., M. Álvarez \& A. DíAz. 2004. Resultados botánicos de la primera expedición científica a la isla Guamblin (Archipiélago de Los Chonos, XI Región, Chile). Revista Geográfica de Valparaíso 35: 225-242.

Ramírez, C., M. Álvarez, A. Díaz \& G. Toledo. 2006. Biodiversidad vegetal de la Isla Ipún en la Reserva Nacional Las Guaitecas (Comuna de Cisnes, XI Región, Chile). Revista Geográfica de Valparaíso 37: 45-66.

Ramírez, C., S. Labbe, C. San Martín \& H. Figueroa. 1990. Sinecología de los bosques de boldo (Peumus boldus) de la cuenca del Río Bueno, Chile. Bosque 11(1): 45-56.

Ramírez, C., C. San Martín, C. Novoa, J. Villagra \& J. Amigo. 2009. Uso de tablas fitosociológicas para detectar especies vegetales con problemas de conservación. Agro Sur 37(2): 91-102.

Ramírez, C., C. San Martín \& P. Ojeda. 1997. Muestreo y tabulación fitosociológica aplicados al estudio de los bosques nativos. Bosque 18(2): 19-27.

Ramírez, C., V. Sandoval, C. San Martín, M. Álvarez, Y. Pérez \& C. NovoA. 2012. El paisaje rural antropogénico de Aisén, Chile: Estructura y dinámica de la vegetación. Gayana Botánica 69(1): 219-231.

Reiche, C. 1898. Flora de Chile. Santiago, Vol. 2: 1-26.

Rodríguez, R. 2011. 93. Anacardiaceae R. Br. En: C. Marticorena y R. Rodríguez (eds.) Flora de Chile 3 (1) Misodendraceae Zygophyllaceae. pp. 88-103. Universidad de Concepción, Chile.

Roeland, K. \& R. Coe. 2005. Tree diversity analysis. A manual and software for common statistical methods for ecological and biodiversity studies. World Agroforestry Centre (ICRAF), Nairobi. 196 pp.

RoIG, F.A. 1994. La vegetación de la Patagonia. En: M.N. Correa (ed.). Flora Patagónica I. pp. 48-154. Colección Científica del INTA, Buenos Aires.

Saldivia, P. \& G. Rojas. 2008. Nuevos registros y antecedentes de la Familia Cactaceae para Chile en la Región de Aisén. Gayana Botánica 65(2): 198-208.

San Martín, C., J. Villagra \& C. Novoa. 2009. Comparación de manejos pratenses del centro-sur de Chile utilizando valores bioindicadores de Ellenberg. Gayana Botánica 66(2): 158-170.

Teillier, S. \& C. Marticorena. 2002. Riqueza florística del Parque 
Nacional Laguna San Rafael, XI Región, Chile. Boletín del Museo Nacional de Historia Natural, Chile 51: 43-73.

Venables, W.N. \& B.D. RIPLEY. 2002. Modern applied statistics with S. Missouri Botanical Garden Press, Missouri. 3348 pp.

VIDAL, O. 2007. Flora Torres del Paine - Guía de campo. FantásticoSur FS Editorial, Punta Arenas. 345 pp.

Vidal, O., J.R. Bannister, V. Sandoval, Y. Pérez \& C. Ramírez. 2011. Woodland communities in the Chilean coldtemperate zone (Baker and Pascua basins): Floristic composition and morpho-ecological transition. Gayana Botánica 68(2): 141-154.
Villagrán, C. \& L.F. Hinojosa. 1997. Historia de los bosques del sur de Sudamérica, II: Análisis fitogeográfico. Revista Chilena de Historia Natural 70: 241-267.

Wikum, D. \& G.F. Shanholtzer. 1978. Application of the BraunBlanquet cover-abundance scale for vegetation analysis in land development studies. Environmental Management 2(4): 323-329.

Zuloaga, F., O. Morrone \& M. Belgrano. 2008. Catálogo de las plantas vasculares del Cono Sur. Monograph in Systematic Botany from the Missouri Botanical Garden, Vol. 107. Missouri. U.S.A. 3348 pp.

Recibido: 11.03 .14

Aceptado: 17.08 .15 